\title{
Study of the MEG Background Activity in Alzheimer's Disease Patients with Scaling Analysis Methods
}

\author{
Carlos Gómez, Student Member, IEEE, Roberto Hornero*, Member, IEEE, Daniel Abásolo, Member, \\ IEEE, Alberto Fernández, and Jesús Poza, Member, IEEE
}

\begin{abstract}
Alzheimer's disease (AD) is one of the most prominent neurodegenerative disorders. The aim of this research work is to study the magnetoencephalogram (MEG) background activity in AD patients using two scaling analysis methods: detrended fluctuation analysis $(D F A)$ and backward detrended moving average (BDMA). Both measures have been designed to quantify correlations in noisy and non-stationary signals. Five minutes of recording were acquired with a 148channel whole-head magnetometer in 15 patients with probable AD and 15 control subjects. Both $D F A$ and $B D M A$ exhibited two scaling regions with different slopes. Significant differences between both groups were found in the second region of $D F A$ and in the first region of $B D M A(p<0.01$, Student's $t$-test $)$. Using receiver operating characteristic curves, accuracies of $83.33 \%$ with $D F A$ and of $80 \%$ with $B D M A$ were reached. Our findings show the usefulness of these scaling analysis methods to increase our insight into AD.
\end{abstract}

\section{INTRODUCTION}

$\mathrm{M}$ AGNETOENCEPHALOGRAPHY (MEG) is a non-invasive technique that allows recording the magnetic fields produced by brain activity. It provides an excellent temporal resolution, orders of magnitude better than other methods for measuring cerebral activity, as magnetic resonance imaging, single-photon-emission computed tomography or positronemission tomography [1]. A good spatial resolution can also be achieved due to the large number of sensors. Moreover, the activity in different parts of the brain can be monitored simultaneously with whole-head equipments, such as the magnetometer used in the present study [1]. On the other hand, the magnetic signals generated by the human brain are extremely weak. Thus, SQUID (Superconducting QUantum Interference Device) sensors are necessary to detect them. In addition, MEG signals must be recorded in a magnetically shielded room. Thus, MEG is characterized by limited availability and high equipment cost.

Alzheimer's disease (AD) is a progressive and irreversible brain disorder of unknown aetiology. It is the main cause of dementia in western countries, accounting for $50-60 \%$ of all

Manuscript received April 22, 2009. This work was supported in part by Ministerio de Ciencia e Innovación under project TEC2008-02241. Asterisk indicates corresponding author.

C. Gómez, R. Hornero, D. Abásolo, and J. Poza are with the Biomedical Engineering Group at Department of Signal Theory and Communications, E.T.S. Ingenieros de Telecomunicación, University of Valladolid, Campus Miguel Delibes, 47011 - Valladolid, Spain (e-mail: cargom@tel.uva.es).

A. Fernández is with the Centro de Magnetoencefalografía Dr. PérezModrego, University Complutense of Madrid, Spain. cases [2]. AD affects $1 \%$ of population aged 60-64 years, but the prevalence increases exponentially with age, so around $30 \%$ of people over 85 years suffer from this disease [3]. Additionally, due to the fact that life expectancy has significantly improved in western countries in the last decades, it is expected that the number of people with dementia increase up to 81 million in 2040 [3]. AD is characterized by neuronal loss and the appearance of neuritic plaques containing amyloid- $\beta$-peptide and neurofibrillary tangles [4]. Clinically, this disease manifests as a slowly progressive impairment of mental functions whose course lasts several years prior to death [4]. Usually, AD starts by destroying neurons in parts of the patient's brain that are responsible for storing and retrieving information. Then, it affects the brain areas involved in language and reasoning. Eventually, many other brain regions are atrophied. Thus, $\mathrm{AD}$ patients may wander, be unable to engage in conversation, appear non-responsive, become helpless and need complete care and attention [5]. Although a definite AD diagnosis is only possible by necropsy, a differential diagnosis with other types of dementia and with major depression should be attempted. The differential diagnosis includes medical history studies, physical and neurological evaluation, mental status tests, and neuroimaging techniques.

The electromagnetic brain activity has been researched in the last decades by means of non-linear techniques. Correlation dimension has been widely used to study the brain activity in $\mathrm{AD}$ patients [6, 7]. Nevertheless, reliable estimation of this classical measure requires a large number of data points and stationary and noise-free time series [8]. As these problems cannot be solved for physiological signals, other non-linear methods are necessary to study brain recordings. In fact, Lempel-Ziv complexity [9-11], Higuchi's fractal dimension [12], approximate entropy [11], sample entropy [10], synchronization likelihood [13], phase lag index [14], and auto-mutual information [15] have been already used to analyze the MEG activity in AD.

In this preliminary study, we have examined the MEG background activity in patients with probable $\mathrm{AD}$ and in agematched control subjects using two scaling analysis measures: detrended fluctuation analysis (DFA) and backward detrended moving average (BDMA). Our purpose is to test the hypothesis that the neuronal dysfunction in $\mathrm{AD}$ is associated with differences in the dynamical processes underlying the MEG recording. 


\section{MATERIAL AND METHODS}

\section{A. Subjects and MEG recording}

The signals were recorded using a 148-channel wholehead magnetometer (MAGNES $2500 \mathrm{WH}$ ) located in a magnetically shielded room. The subjects lay comfortably on a patient bed, in a relaxed state and with their eyes closed. They were asked to stay awake and to avoid eye and head movements. For each subject, five minutes of recording were acquired at a sampling frequency of $678.17 \mathrm{~Hz}$. These recordings were down-sampled by a factor of four, obtaining a sampling rate of $169.55 \mathrm{~Hz}$. Data were digitally filtered between 0.5 and $40 \mathrm{~Hz}$. Finally, artifact-free epochs of 5 seconds (848 samples) were selected.

MEG data were acquired from 30 subjects. Cognitive status was screened in both groups with the Mini Mental State Examination (MMSE). The AD group consisted of fifteen patients ( 5 men and 10 women; age = $72.33 \pm 9.04$ years, mean \pm standard deviation, SD) fulfilling the criteria of probable $\mathrm{AD}$, according to the criteria of the National Institute of Neurological and Communicative Disorders and Stroke - Alzheimer's and Related Disorders Association. The mean MMSE score for the patients was $17.67 \pm 3.94$ points. Patients were free of other significant medical, neurological and psychiatric diseases than AD. Moreover, any of the participants in the study used medication that could be expected to influence in the MEG recording.

The control group consisted of fifteen control subjects without past or present neurological disorders ( 7 men and 8 women; age $=72.53 \pm 5.40$ years, MMSE score $=29.00 \pm$ 0.33 points). All control subjects and all caregivers of the patients gave their informed consent for the participation in the current study. The local Ethics Committee approved this study.

\section{B. Detrended Fluctuation Analysis (DFA)}

$D F A$ is a measure widely used for the detection of longrange correlations and fluctuations in time series [16]. This method provides a simple quantitative parameter (the scaling exponent) to represent the correlation properties of a signal [17]. DFA permits the detection of long-range correlations embedded in seemingly non-stationary time series. Additionally, it avoids the spurious detection of apparent long-range correlations that are an artifact of non-stationarity [17]. Given a one dimensional time series $X=x_{1}, x_{2}, \ldots, x_{N}$, we describe the algorithm to compute the $D F A[16,18]$ :

1) Integrate the signal $X$ :

$$
y_{i}=\sum_{j=1}^{i}\left[x_{j}-\bar{X}\right]
$$

where $\bar{X}$ represents the mean value of $X$.

2) The integrated time series $Y$ is divided into windows of equal size $k$. The size of the windows is ranged between 3 and 84, as one-tenth of the signal length can be considered as the maximum window size when using DFA [17].
3) Within each window, labeled $b(b=1,2, \ldots, B)$, perform a least-square fit of $Y$ by a straight line $\bar{Y}^{b}$. This is the semilocal trend for the $b^{\text {th }}$ window.

4) Define $F_{b}^{2}(k)$ to be the variance of the fluctuation $Y$ from $\bar{Y}^{b}$ in the $b^{\text {th }}$ window:

$$
F_{b}^{2}(k)=\frac{1}{k} \sum_{t=(b-1) k+1}^{b k}\left(y_{t}-\bar{y}_{t}^{b}\right)^{2}
$$

5) The square root of the average of $F_{b}^{2}(k)$ over all windows is the rms fluctuation from the semilocal trends in $B$ windows, each having $k$ time points:

$$
F(k)=\sqrt{\frac{1}{B} \sum_{b=1}^{B} F_{b}^{2}(k)}
$$

6) Finally, the study of the dependence of $F(k)$ on the window size $k$ is the essence of $D F A$. If it is a power-law behavior $F(k) \propto k^{\alpha}$, the scaling exponent is an indicator of the nature of the fluctuations in the MEG signals.

\section{Backward Detrended Moving Average (BDMA)}

The detrended moving average (DMA) method is a new approach to quantify correlation properties in non-stationary signals with underlying trends [19]. Serletis and Rosenberg [20] suggested that DMA is an improvement over DFA. $D M A$ does not require dividing the series into nonoverlapping windows. Instead, the DMA method detrends the series by subtracting a continuous function, the moving average. Additionally, $D M A$ is more accurate since the moving average is a better low-pass filter when compared to the polynomial filter used for $D F A$ [20]. In this study, we have used the category of $D M A$ called $B D M A$ [21]. To perform the $B D M A$ of a time series $X=x_{1}, x_{2}, \ldots, x_{N}$, we pursue the following steps [21]:

1) The signal $X$ is integrated:

$$
y_{i}=\sum_{j=1}^{i}\left[x_{j}-\bar{X}\right]
$$

where $\bar{X}$ represents the mean value of $X$.

2) The second step of the BDMA is to detect trends in data employing a moving average. For a window of size $k$, the backward moving average is defined as:

$$
\widetilde{y}_{i}^{k}=\frac{1}{k} \sum_{n=0}^{k-1} y_{i-n}
$$

3) Afterwards, the signal is detrended by substracting the trend $\tilde{y}^{k}$ from the integrated profile $Y$ :

$$
C_{i}^{k}=y_{i}-\tilde{y}_{i}^{k}
$$

4) Then calculate the fluctuation for a window of size $k$ as:

$$
F(k)=\sqrt{\frac{1}{N-k+1} \sum_{i=k}^{K}\left(C_{i}^{k}\right)^{2}}
$$

5) As in DFA method, a power law relation between the fluctuation function $F(k)$ and the scale $k$ indicates a selfsimilar behavior. 


\section{RESULTS}

DFA method was applied for the 148 MEG channels using window sizes between 3 and 84 samples. To simplify the analyses, the results were averaged over all channels. $F(k)$ as a function of $k$ was plotted in a $\log \log$ scale, as Fig. 1 illustrates. If the plot displays a linear scaling region with a certain scaling exponent, then there is a power-law behavior in the time series [22]. Our results showed two scaling regions with different slopes: $\alpha_{1}$ for $3 \leq k \leq 7$, and $\alpha_{2}$ for $14 \leq$ $k \leq 73$. No significant differences were found between the $\alpha_{1}$ values of $\mathrm{AD}$ patients and control subjects $(p=0.7107>$ 0.01 ; Student's $t$-test). On the other hand, the differences between both groups were statistically significant when the slopes of the second scaling region were analyzed ( $p=$ $0.0015<0.01$; Student's $t$-test).

We next apply the BDMA method to the MEG recordings with windows sizes between 2 and 84 samples. BDMA profiles were obtained representing the natural logarithm of $F(k)$ versus the natural logarithm of $k$ (see Fig. 2). This scaling analysis method also showed two scaling regions, $\alpha_{1}$ and $\alpha_{2}$. Significant differences between both groups were found with $\alpha_{1}(p=0.0001)$, whereas the $p$-value obtained with $\alpha_{2}$ was 0.1065 .

Finally, receiver operating characteristic (ROC) curves were used to assess the ability of these measures to discriminate $\mathrm{AD}$ patients from controls. This method summarizes the performance of a two-class classifier across the range of possible thresholds. Fig. 3 and 4 represent the ROC curves obtained at both scaling regions with $D F A$ and $B D M A$, respectively. The highest accuracy was achieved when the slope $\alpha_{2}$ of $D F A$ was analyzed: $83.33 \%$. In the first scale region of $B D M A$, we achieved the highest value of area under the ROC curve (AROC): 0.8667. Table I shows the sensitivity, specificity, accuracy and AROC values obtained with $D F A$ and $B D M A$ in each scaling region.

\section{DISCUSSION AND CONCLUSION}

We analyzed the MEG background activity from 15 patients with probable $\mathrm{AD}$ and 15 age-matched control subjects by means of $D F A$ and $B D M A$ methods. Our purpose was to check the hypothesis that MEG background activity was different in $\mathrm{AD}$ patients than in control subjects. Both measures have proven to be effective in discriminating $\mathrm{AD}$ patients from controls. Our results revealed that $\alpha_{2}$ values of $D F A$ were significantly lower for the controls than for the $\mathrm{AD}$ patients. On the other hand, significant differences between both groups appear in the first scaling region of $B D M A$. Because both groups were carefully matched for age, these changes in the fluctuations of MEG signals may well represent the cognitive dysfunction in AD. Additionally, accuracies of $83.33 \%$ and $80 \%$ were reached with $D F A$ and $B D M A$ by means of ROC curves.

Other non-linear methods have been already used to study the MEG activity in AD. In [9-11], Lempel-Ziv complexity

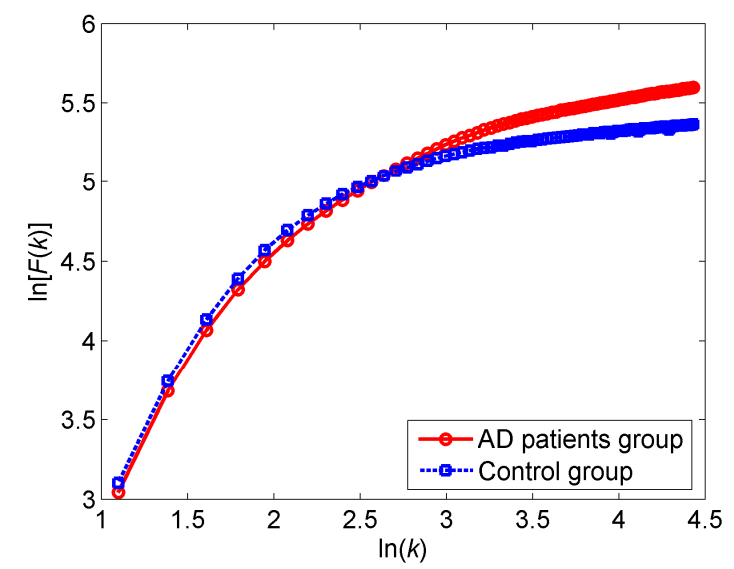

Fig. 1. DFA profiles, $F(k)$ versus $k$ in a log-log scale, for AD patients and control groups.

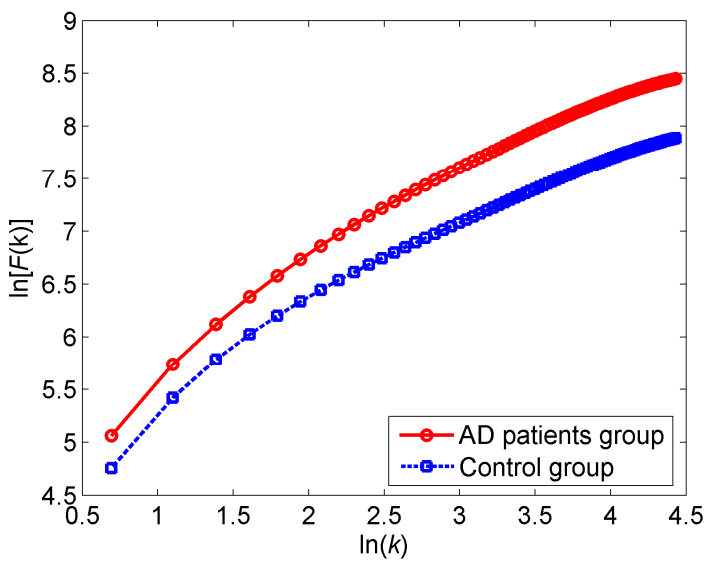

Fig. 2. $B D M A$ profiles, $F(k)$ versus $k$ in a $\log -\log$ scale, for $\mathrm{AD}$ patients and control groups.

TABLE I

SENSITIVITY, SPECIFICITY, ACCURACY AND AROC VALUES OBTAINED WITH DFA AND BDMA

\begin{tabular}{lcccc}
\hline \hline & Sensitivity & Specificity & Acurracy & AROC \\
\hline \hline$\alpha_{1}$ of $D F A$ & $60.00 \%$ & $66.67 \%$ & $63.33 \%$ & 0.5511 \\
$\alpha_{2}$ of $D F A$ & $86.67 \%$ & $80.00 \%$ & $83.33 \%$ & 0.8400 \\
$\alpha_{1}$ of $B D M A$ & $80.00 \%$ & $80.00 \%$ & $80.00 \%$ & 0.8667 \\
$\alpha_{2}$ of $B D M A$ & $60.00 \%$ & $86.67 \%$ & $73.33 \%$ & 0.6711 \\
\hline \hline
\end{tabular}

values were significantly lower in the recordings from $\mathrm{AD}$ patients than in those obtained from control subjects. This complexity loss in AD was confirmed using the algorithm of fractal dimension proposed by Higuchi [12]. Nevertheless, van Cappellen van Walsum et al. [7] suggested that this decreased complexity in the MEG background activity of $\mathrm{AD}$ patients may appear only in the low frequency bands. Other MEG studies revealed that brains affected by AD show a more regular physiological behaviour [10, 11]. Stam et al. [13] found changes of long and short distances 


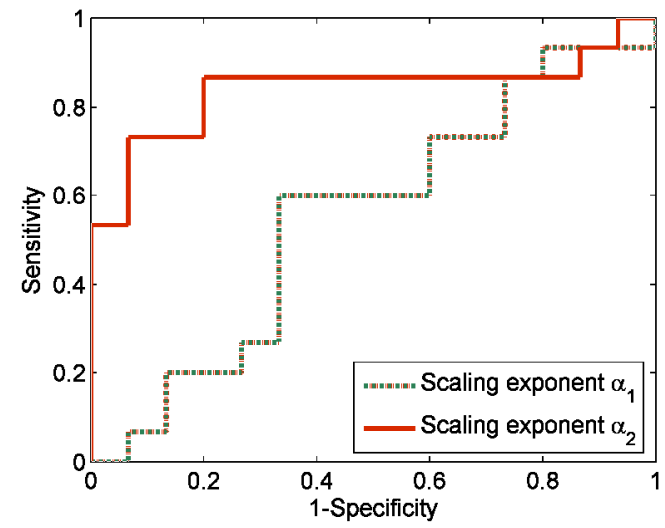

Fig. 3. ROC curves showing the discrimination between $\mathrm{AD}$ patients and control subjects with $D F A$.

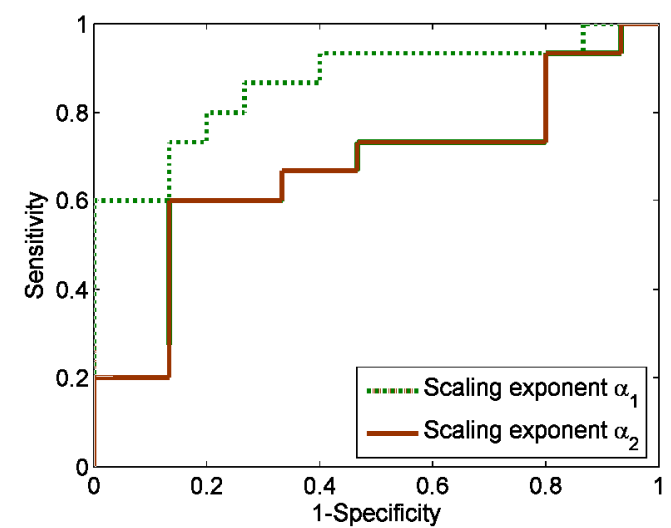

Fig. 4. ROC curves showing the discrimination between AD patients and control subjects with BDMA.

interaction in the frequency bands theta, alpha1, beta and gamma using the synchronization likelihood. Finally, AD patients have shown a decrease in the mean values of phase lag index in the lower alpha and beta bands [14].

Some limitations of our study merit consideration. Firstly, the sample size is small. Thus, a larger database is needed to confirm our results. Secondly, our results do not show if these measures can detect a gradation of the disease process. Therefore, future efforts will be addressed to characterize MEG background activity in AD and in other pathologies. It is particularly interesting to study MEGs from patients with mild cognitive impairment, since several authors have considered this disease as a prodromal phase of AD.

To sum up, our results suggest that both $D F A$ and $B D M A$ are appropriate methods to differentiate $A D$ patients from elderly controls. Nevertheless, this is a preliminary study and a larger database is needed to confirm our results.

\section{REFERENCES}

[1] R. Hari, "Magnetoencephalography in clinical neurophysiological assessment of human cortical functions," in Electroencephalography: basic principles, clinical applications, and related fields, 5th ed., E. Niedermeyer, and F. Lopes da Silva, Eds. Philadelphia: Lippincontt Williams \& Wilkins, 2005, pp. 1165-1197.

[2] K. Blennow, M. J. de Leon, and H. Zetterberg, "Alzheimer's disease," Lancet, vol. 368, pp. 387-403, 2006.
[3] A. F. Jorm, "Cross-national comparisons of the occurrence of Alzheimer's and vascular dementias," Eur. Arch. Psychiatry Clin. Neurosci., vol. 240, pp. 218-222, 1991.

[4] J. Jeong, "EEG dynamics in patients with Alzheimer's disease," Clin. Neurophysiol., vol. 115, pp. 1490-1505, 2004.

[5] J. L. Cummings, "Alzheimer's disease," N. Engl. J. Med., vol. 351, pp. 56-67, 2004

[6] J. Jeong, S. J. Kim, and S. H. Han, "Non-linear dynamical analysis of the EEG in Alzheimer's disease with optimal embedding dimension," Electroencephalogr. Clin. Neurophysiol., vol. 106, pp. 220-228, 1998.

[7] A. M. van Cappellen van Walsum, Y. A. L. Pijnenburg, H. W. Berendse, B. W. van Dijk, D. L. Knol, Ph. Scheltens, and C. J Stam, "A neural complexity measure applied to MEG data in Alzheimer's disease," Clin. Neurophysiol., vol. 114, pp. 1034-1040, 2003.

[8] J. P. Eckmann and D. Ruelle, "Fundamental limitations for estimating dimensions and Lyapunov exponents in dynamical systems," Physica $D$, vol. 56, pp. 185-187, 1992.

[9] C. Gómez, R. Hornero, D. Abásolo, A. Fernández, and M. López, "Complexity analysis of the magnetoencephalogram background activity in Alzheimer's disease patients," Med. Eng. Phys., vol. 28, pp. 851-859, 2006.

[10] C. Gómez, R. Hornero, D. Abásolo, A. Fernández, and J. Escudero, "Analysis of MEG background activity in Alzheimer's disease using non-linear methods and ANFIS," Ann. Biomed. Eng., vol. 37, pp. 586-594, 2009.

[11] R. Hornero, J. Escudero, A. Fernández, J. Poza, and C. Gómez, "Spectral and non-linear analyses of MEG background activity in patients with Alzheimer's disease," IEEE Trans. Biomed. Eng., vol. 55, pp. 1658-1665, 2007.

[12] C. Gómez, A. Mediavilla, R. Hornero, D. Abásolo, and A. Fernández, "Use of the Higuchi's fractal dimension for the analysis of MEG recordings from Alzheimer's disease patients," Med. Eng. Phys., vol. 31, pp. 306-313, 2009.

[13] C. J. Stam, B. F. Jones, I. Manshanden, A. M. van Cappellen van Walsum, T. Montez, J. P. A. Verbunt, J. C. de Munck, B. W. van Dijk, H. W. Berendse, and P. Scheltens, "Magnetoencephalographic evaluation of resting-state functional connectivity in Alzheimer's disease," Neuroimage, vol. 32, pp. 1335-1344, 2006.

[14] C. J. Stam, W. de Haan, A. Daffertshofer, B. F. Jones, I. Manshanden, A. M. van Cappellen van Walsum, T. Montez, J. P. A. Verbunt, J. C. de Munck, B. W. van Dijk, H. W. Berendse, and P. Scheltens, "Graph theoretical analysis of magnetoencephalographic functional connectivity in Alzheimer's disease," Brain, vol. 132, pp. 213-224, 2009.

[15] C. Gómez, R. Hornero, D. Abásolo, A. Fernández, and J. Escudero, "Analysis of the magnetoencephalogram background activity in Alzheimer's disease patients with auto-mutual information," Comput. Meth. Programs Biomed., vol. 87, pp. 239-247, 2007.

[16] C. K. Peng, S. V. Buldyrev, S. Havlin, M. Simons, H. E. Stanley, and A. L Goldberger, "Mosaic organization of DNA nucleotides," Phys. Rev. E, vol. 49, pp. 1685-1689, 1994.

[17] K. Hu, P. C. Ivanov, Z. Chen, P. Carpena, and H. E. Stanley, "Effects of trends on detrended fluctuation analysis," Phys. Rev. E, vol. 64, pp. 011114,2001

[18] R. C. Hwa and T. C. Ferree, "Scaling properties of fluctuations in the human electroencephalogram," Phys. Rev. E, vol. 66, pp. 021901, 2002.

[19] E. Alessio, A. Carbone, G. Castelli, and V. Frappietro, "Second-order moving average and scaling of stochastic time series," Eur. Phys. J. $B$, vol. 27, pp. 197-200, 2002.

[20] A. Serletis and A. A. Rosenberg, "The Hurst exponent in energy futures prices," Physica A, vol. 380, pp. 325-332, 2007.

[21] L. Xu, P. Ch. Ivanov, K. Hu, Z. Chen, A. Carbone, and H. E. Stanley, "Quantifying signals with power-law correlations: A comparative study of detrended fluctuation analysis and detrended moving average techniques," Phys. Rev. E, vol. 71, pp. 051101, 2005.

[22] D. Abásolo, R. Hornero, J. Escudero, and P. Espino, "A study of the possible usefulness of detrended fluctuation analysis of the electroencephalogram background activity in Alzheimer's disease," IEEE Trans. Biomed. Eng., vol. 55, pp. 2171-2179, 2008. 